\title{
Effectiveness of Standalone Weight-bearing Radiographs in Guiding the Management of Supination External Rotation Type Isolated Lateral Malleolar Fractures in Indian Population: A Prospective Clinical Study
}

\author{
Singaravadivelu Vaidyanathan ${ }^{1}$, Vignesh Murali ${ }^{2}$, Aju Bosco ${ }^{3}$
}

\begin{abstract}
Aim and objective: To assess the effectiveness of standalone weight-bearing radiographs (WBR) in guiding the management of Supination External Rotation (SER), type of isolated lateral malleolar fractures in Indian population.

Materials and methods: In a prospective study involving 19 consecutive patients with SER type of isolated lateral malleolar fractures, stability of the ankle was assessed using both WBR and external rotation stress radiographs. The decision toward conservative or surgical management was made solely on the assessment of ankle stability on WBR alone. Fractures with a medial clear space of $<5 \mathrm{~mm}$ were deemed stable and managed conservatively (full weight-bearing in cast), while unstable ones were managed surgically (with open reduction and fibular plating). Periodic follow-up was done to assess radiological union and functional outcomes using the American Orthopaedic Foot and Ankle Society (AOFAS) score. Patient's pain score visual analogue scale (VAS) and compliance was assessed during radiographic assessment.

Results: Based on WBR alone, 17/19 (89.5\%) fractures were found to be stable and treated conservatively, while 2/19 (10.5\%) fractures were unstable and managed surgically. The mean AOFAS score in the conservative group [92.9 \pm 3.4 (excellent)] and surgical group [91.5 \pm 1.5 (excellent)] were comparable at latest follow-up. Complete radiological union was seen at $8.8 \pm 1.9$ (range, 8-14) weeks in 82\% (14/17), while delayed union (union taking place $>8$ weeks) was seen in three patients in the conservative group. Four patients ( $21 \%$ ), who would have been operated had external rotation stress views been used in decision making, were managed conservatively with successful clinical outcome and excellent AOFAS scores. Patient compliance was good with WBR as compared to external rotation stress radiographs.

Conclusion: Standalone WBR are feasible, and reliable in detecting injury of the deep deltoid ligament and the consequent instability of the tibiotalar joint, in Weber type B fractures. They are associated with better patient compliance.

Clinical significance: Standalone WBR serve as a useful guide in decision making between conservative and surgical treatment, in SER type isolated lateral malleolar fractures.

Keywords: Ankle, Ankle fracture, Ankle instability, Deltoid ligament, Lateral malleolus, Weight-bearing radiograph.

Journal of Orthopedics and Joint Surgery (2022): 10.5005/jp-journals-10079-1084
\end{abstract}

\section{INTRODUCTION}

Fractures of the ankle constitute about $10 \%$ of all fractures. ${ }^{1,2}$ Isolated lateral malleolar fractures (Weber type B) resulting from Supination External Rotation (SER) injuries, are the most common ankle fractures. ${ }^{2-4}$ These fractures can be managed either conservatively or surgically depending on the stability of the ankle mortise. ${ }^{5}$ About $20 \%$ of these fractures which are surgically treated, ${ }^{6}$ have been plagued by complications which include infection, damage to superficial peroneal nerve, ${ }^{7}$ prominent hardware requiring removal, ${ }^{8}$ peroneal tendon complications among many others.

Nonoperative management of Weber type B fractures with a stable congruent ankle mortise denoting an intact deltoid ligament, gives good to excellent long-term outcomes. ${ }^{4-6}$ It is critical to diagnose fractures with associated ankle joint instability [due to a critical rupture of the deep deltoid ligament (DDL)] as nonoperative management of these unstable fractures can lead to poor functional outcomes. ${ }^{4,5,9,10}$ Identifying the degree of rupture of DDL based on clinical findings (tenderness, ecchymosis and swelling over medial malleolus) alone is associated with poor sensitivity and specificity. ${ }^{11,12}$

\footnotetext{
1,2Institute of Orthopedics and Traumatology, Madras Medical College, Chennai, Tamil Nadu, India

${ }^{3}$ Orthopedic Spine Surgery Unit, Institute of Orthopedics and Traumatology, Madras Medical College, Chennai, Tamil Nadu, India

Corresponding Author: Aju Bosco, Orthopedic Spine Surgery Unit, Institute of Orthopedics and Traumatology, Madras Medical College, Chennai, Tamil Nadu, India, Phone: +91 9443456190, e-mail: ajubosco@gmail.com
}

How to cite this article: Vaidyanathan S, Murali V, Bosco A Effectiveness of Standalone Weight-bearing Radiographs in Guiding the Management of Supination External Rotation Type Isolated Lateral Malleolar Fractures in Indian Population: A Prospective Clinical Study. J Orth Joint Surg 2022;4(1):29-34.

Source of support: $\mathrm{Nil}$

Conflict of interest: None

Existing literature describes different radiological methods to identify the integrity of the DDL while subjecting the ankle joint to manual or gravity-assisted stress. ${ }^{8,13}$ These methods, tend to overestimate unstable fractures, that resulted in 
a greater number of stable fractures being subjected to operative treatment. ${ }^{8,13}$

Weight-bearing radiographs (WBR) of the ankle mortise were introduced to evaluate DDL integrity, as weight-bearing represents the physiological loading of the ankle joint. The ankle joint is tested functionally in comparison with other radiographic methods (Table 1). ${ }^{14-16}$

The study aims to assess the reliability of standalone WBR of the ankle in differentiating stable from unstable isolated lateral malleolar fractures in the Indian population, thereby guiding the management of these fractures.

\section{Materials and Methods}

This is a prospective study including patients with unilateral closed Weber type B and Lauge Hansen SER type of isolated fractures of lateral malleolus between February 2018 and October 2019 at our Institution. Approval from the Institutional Ethics Committee was obtained before starting the study. Informed consent was obtained from all participants.

All 19 patients were included after their initial postinjury ankle radiographs showed only fracture of lateral malleolus. Open fractures, bilateral injuries, those with fractures of the medial or posterior malleolus, skeletally immaturity, concomitant medical, or orthopedic comorbidities that prevented weight-bearing of the affected ankle were excluded.

\section{Study Protocol}

All patients with isolated lateral malleolar fractures were immobilized in a below knee plaster of paris slab. Participants were given analgesics and kept on non-weight-bearing along with limb elevation during the initial 7-10 days. On the 8th day, the slab was removed and radiographs of the injured ankle (anteroposterior, mortise, and lateral views) were taken with ankle subjected to both weight-bearing and external rotation stress, the Medial Clear Space (MCS) was measured using integrated software of the digital radiographic system. Other measurements like superior clear space, tibiofibular overlap, tibiofibular clear space, and talo-crural angle were measured in weight-bearing mortise view of ankle. The external rotation stress radiographs were taken by applying external rotation force on dorsiflexed ankle for all patients by the same orthopedic surgeon to ensure reproducibility in method of testing (Fig. 1). The medial and superior clear space were assessed in all patients. WBR were obtained after ensuring that the patient was able to comfortably bear weight on both legs without pain (Fig. 2). Ankle joint stability was assessed solely based

Table 1: A comparison of stability of the ankle joint assessed by weight-bearing radiographs and external rotation stress radiographs of ankle joint

\begin{tabular}{lcc}
\hline Radiographic criteria & $\begin{array}{c}\text { External rotation } \\
\text { stress view } \\
\text { stable }(\mathrm{MCS}<5 \mathrm{~mm})\end{array}$ & $\begin{array}{c}\text { External rotation } \\
\text { stress view unstable } \\
(\mathrm{MCS}>5 \mathrm{~mm})\end{array}$ \\
\hline $\begin{array}{l}\text { Weight-bearing } \\
\text { radiograph stable }\end{array}$ & $13(68 \%)$ & $4(21 \%)$ \\
$(\mathrm{MCS}<5 \mathrm{~mm})$ & & \\
$\begin{array}{l}\text { Weight-bearing } \\
\text { radiograph unstable } \\
(\mathrm{MCS}>5 \mathrm{~mm})\end{array}$ & 0 & $2(11 \%)$ \\
\hline
\end{tabular}

on the weight-bearing ankle mortise radiograph as elucidated below (Fig. 3).

While on conservative management, patients were allowed full weight-bearing and were asked to consult if they developed pain/swelling or breakage of the plaster cast. Anteroposterior radiographs, mortise views, and lateral radiographs of the ankle were obtained at each follow-up visit. After confirming evidence of union on radiographs, follow-up was done bimonthly for 6-8 months.

After 6-8 months, the patients were instructed to report if they experienced pain or discomfort. Clinical and functional outcomes were assessed using American Orthopaedic Foot and Ankle Society (AOFAS) score after assessment of radiographic union and after cast removal. Radiographic evaluation for any residual instability was done by measurement of the medial clear space and evidence of late talar shift. Surgically treated patients were followed up every month to assess fracture union on radiographs. AOFAS score was assessed at each follow-up visit from the 3rd month after surgery. Patients were followed up to detect complications, discomfort during activity and any new functional impairment.

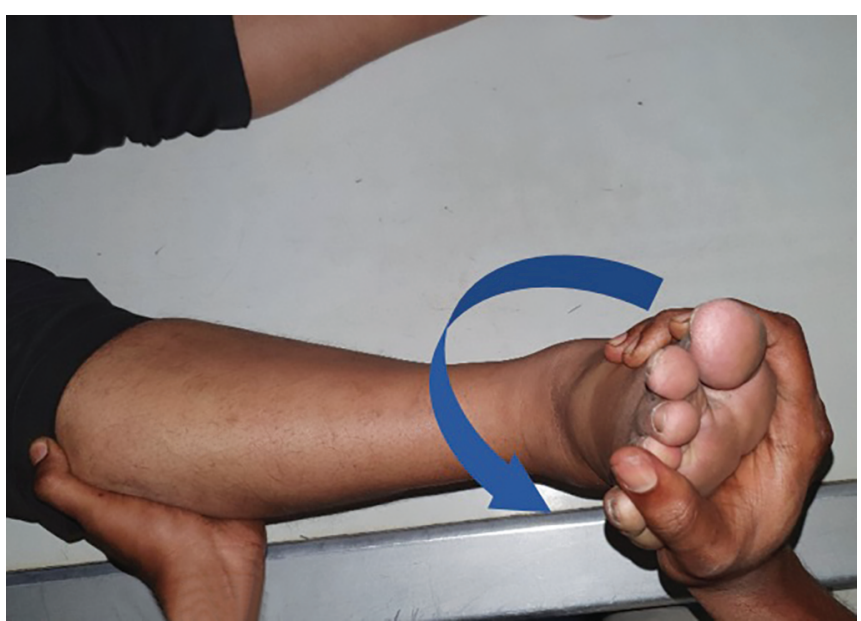

Fig. 1: Method of applying stress to the ankle joint in external rotation stress view

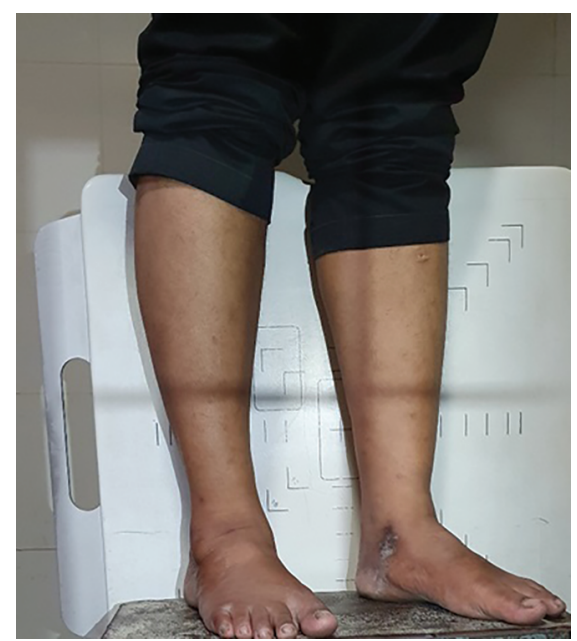

Fig. 2: Weight-bearing radiograph 


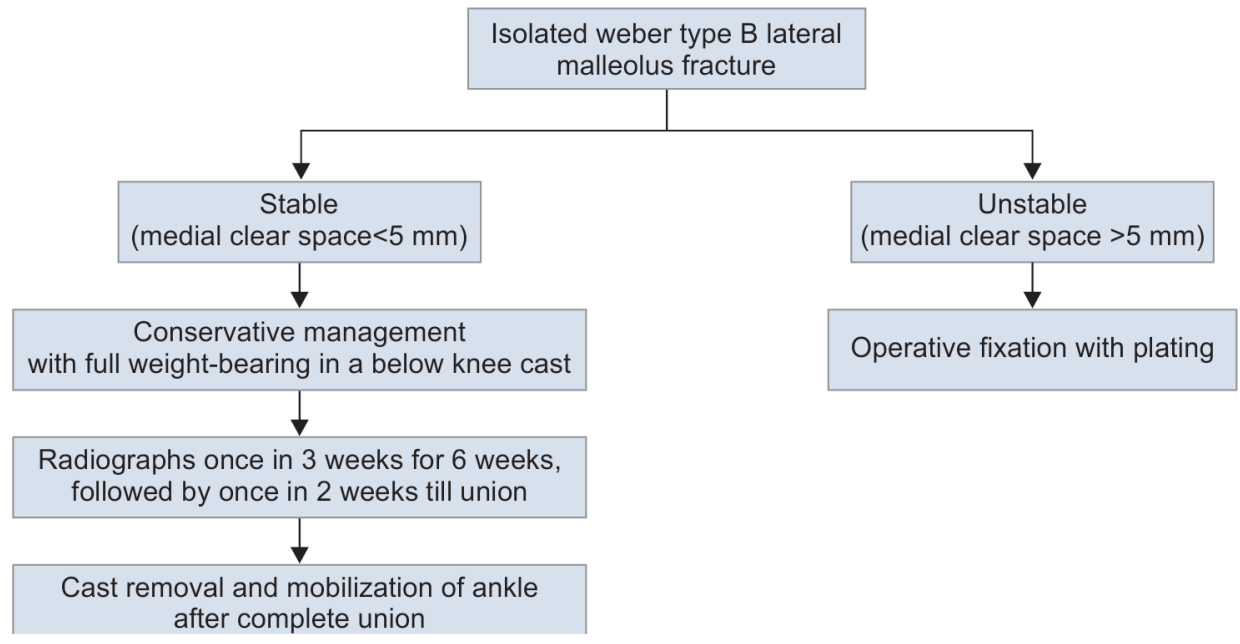

Fig. 3: Management protocol of treating Weber type B isolated lateral malleolar fractures
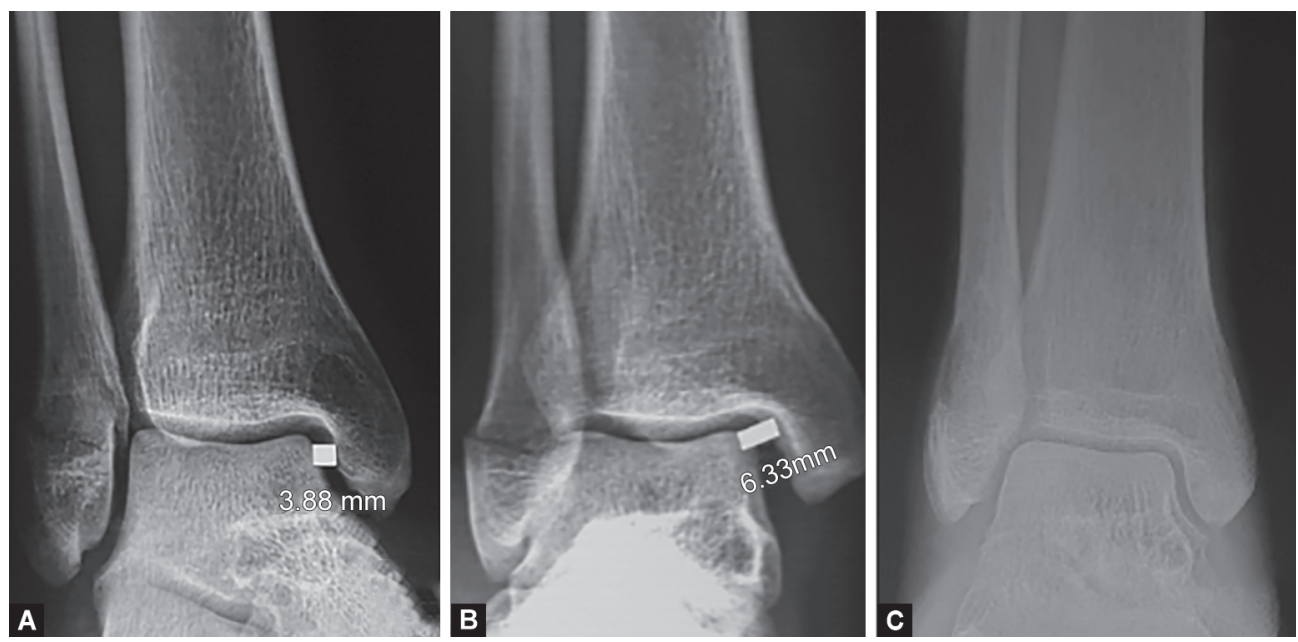

Figs 4A to C: (A) Medial clear space measurements in weight-bearing view; (B) External rotation stress view; (C) Ankle radiograph taken at 8 weeks follow-up after conservatively treating the patient as guided by medial clear space measurements on weight-bearing radiograph of ankle

\section{Results}

Nineteen patients (13 males, 6 females) with isolated lateral malleolar fractures were included in the study. All fractures were SER injuries with Weber type B (Syndesmotic) fracture of fibula. The mean age at presentation was $44.2 \pm 11.4$ years (range, 23-68 years). The most affected age group in our study was $40-50$ years (32\%) followed by $30-40$ years $(26 \%)$.

While evaluating the 19 patients with WBR, 17 (89\%) had stable fracture pattern with medial clear space $<5 \mathrm{~mm}$, lateral talar shift $<1 \mathrm{~mm}$, superior clear space of $<1 \mathrm{~mm}$. Two (11\%) had unstable fracture pattern with DDL injury as evidenced by a medial clear space of $>5 \mathrm{~mm}$ along with a lateral talar shift of $>1 \mathrm{~mm}$. Four (21\%) fractures were found to be stable on WBR but unstable on external rotation stress view. These were also treated conservatively (Fig. 4).

Patients were followed up for a minimum of 1 year with a mean follow-up of $13.9 \pm 1.23$ (range, 12-16) months. None of the patients in the stable group who were conservatively managed, developed ankle instability requiring surgical fixation of the fracture or any adverse event in the interim. No patient complained of any major activity related discomfort or impairment of function. The two patients treated surgically with plating (Fig. 5) had no adverse effects related to plating like wound complication, infection, and peroneal tendon irritation requiring plate removal.

All fractures treated conservatively achieved radiological union at a mean of at $8.8 \pm 1.9$ (range, $8-14$ ) weeks. In the present study, surgically treated fractures showed evidence of radiological union by $10-12$ weeks. Delayed union, in our study is defined as radiological union taking more than the expected 8 weeks of conservative management. Delayed union was observed in three patients, with one patient showing complete union by 10 weeks, and two by 14 weeks. Remaining 14 patients (82\%) managed conservatively achieved radiological union by 8 weeks.

One patient treated conservatively had residual medial clear space of $4.8 \mathrm{~mm}$ and talar shift of $1 \mathrm{~mm}$ with no tibiofibular overlap, but she was asymptomatic and able to perform her activities routinely with AOFAS score of 88 in her last follow-up at 14 months. No patients had nonunion, arthritis, or plaster of paris related complication in our study. No patient treated conservatively by our protocol had delayed increase in medical clear space of $>5 \mathrm{~mm}$ nor persistent discomfort or functional impairment requiring surgery.

American Orthopaedic Foot and Ankle Society score was used to assess the functional outcomes (Table 2). The mean AOFAS score in the conservative group $[92.9 \pm 3.4$ (excellent)] and 

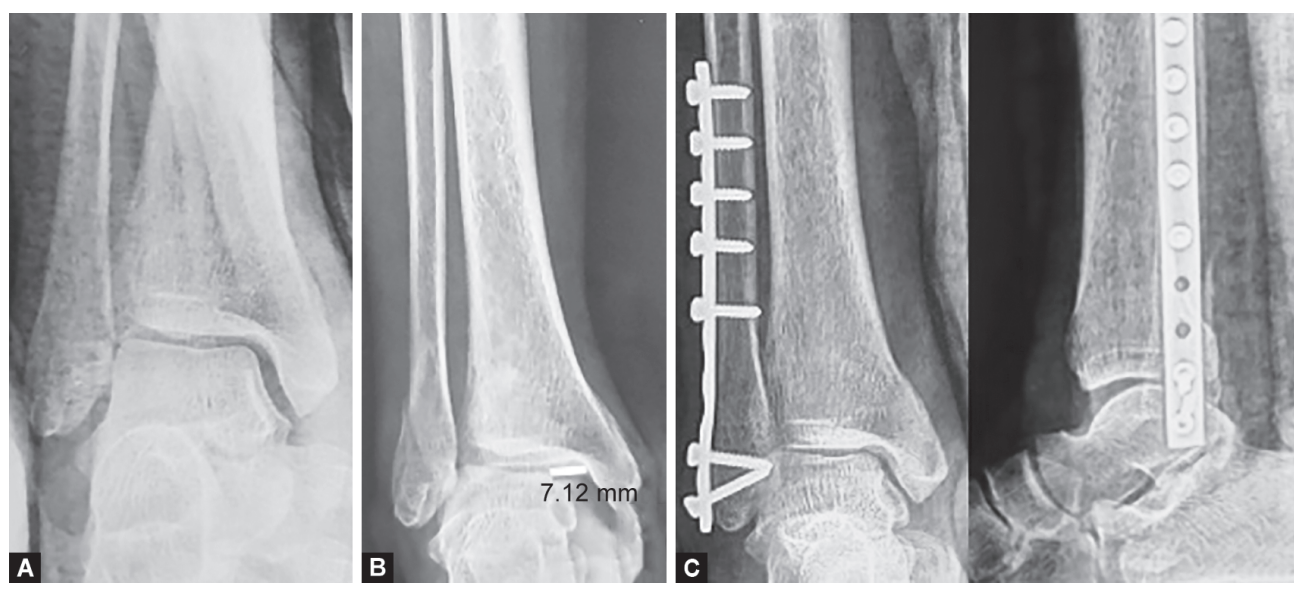

Figs 5A to C: (A) Initial post trauma radiograph; (B) Weight-bearing radiograph with MCS measurement; (C) Postoperative radiographs of 54-year-old patient with isolated lateral malleolar fracture

Table 2: Functional outcome analysis of conservatively managed patients

\begin{tabular}{|c|c|c|c|c|}
\hline \multirow[b]{2}{*}{ S. No. } & \multirow[b]{2}{*}{ AOFAS scores } & \multicolumn{2}{|c|}{ Number of patients } & \multirow[b]{2}{*}{ Percentage } \\
\hline & & Views & Number & \\
\hline \multirow[t]{2}{*}{1} & Excellent $(>90)$ & Both views stable & 10 & 76 \\
\hline & & Only WB view stable & 3 & \\
\hline \multirow[t]{2}{*}{2} & Good (80-89) & Both views stable & 3 & 24 \\
\hline & & Only WB view stable & 1 & \\
\hline 3 & Fair (70-79) & & & 0 \\
\hline 4 & Poor $(<69)$ & & & 0 \\
\hline
\end{tabular}

OOFAS, American Orthopedic Foot and Ankle Society scores

surgical group [91.5 \pm 1.5 (excellent)] were comparable at latest follow-up. The four (21\%) fractures, which were found to be stable on WBR but unstable on stress views, had an excellent AOFAS score of 93.

The two patients managed surgically with plating for fibula had excellent AOFAS scores of 90 and 93 at the end of 14-month follow-up. The mean visual analogue scale (VAS) scores for pain were significantly less with WBR $(6.2 \pm 0.8)$ as compared to external rotation stress radiographs $(8.1 \pm 0.7)$.

\section{Discussion}

Stability of the ankle mortise is the key factor which determines the modality of treatment (conservative or surgical) in Weber type $B$, isolated lateral malleolar fractures (SER injuries)..$^{5}$ Stable fractures without deltoid ligament injury can be managed successfully without surgical intervention. The presence of an associated deltoid ligament injury which cannot be identified in radiographs as easily as the fracture itself, can make these seemingly stable fractures, unstable.

Patients' ability to withstand pain while stressing the ankle joint, the need for anesthesia and personnel to apply the stress while taking the radiograph, as well as concern regarding the correct method of application of stress were the disadvantages of using manual stress radiographs for assessment of ankle instability. ${ }^{15}$ Published studies have shown that manual stress radiographs tend to overestimate complete deltoid ligament rupture and the need for surgery. ${ }^{13,14}$

The gravity stress view proved equally efficacious as external rotation stress view in detecting instability with advantage of better reproducibility, not requiring a person to apply stress and thereby eliminating the bias arising due to variation in stress applied to each patient. ${ }^{17}$ Though simple to perform, it was plagued by the same complication of overestimation of unstable fractures and lack of control over position of foot in sagittal plane at the time of taking radiograph. ${ }^{17,18}$

In this study, only two (11\%) patients were diagnosed with ankle instability, solely based on assessment by WBR. Similar observations were made by Weber et al.,15 (10\% unstable fractures) and Seidel et al., ${ }^{18}$ (13\% unstable fractures). Had external rotation stress views been used for assessment of ankle instability, four (21\%) fractures would have been diagnosed as unstable and treated surgically. The incidence of unstable fractures was even higher with the use of gravity stress view $(55 \%)^{18}$ and external rotation stress view $(37 \%)^{19}$ for DDL injury in isolated lateral malleolar fractures.

In our study, the sensitivity of external rotation stress view in detecting instability was found to be $100 \%$ but the specificity was only $76 \%$ with poor positive predictive value of only $33 \%$. The other parameters used for conservative management such as tibiofibular overlap $(>1 \mathrm{~mm})$ and tibiofibular clear Space $(<5 \mathrm{~mm})$ were not accurate in detecting instability.

In the study by Dawe et al., there is a tenfold increase in propensity to fix isolated lateral malleolus fractures if gravity stress view alone is taken to indicate instability. ${ }^{8}$ These findings correlated with our study where an additional $4(21 \%)$ of fractures would have been categorized as unstable. These patients might have undergone operative intervention, had WBR not been used. These patients might have had partial rupture of deltoid ligament which does not warrant surgery. Thus weight-bearing 
during conservative management provides much needed functional stability despite the presence of a partial DDL tear.

The incidence of complications associated with surgery ranges from $10-20 \%$. $78,20-22$ Infection and poor wound healing were the most common complications, both related to the subcutaneous position of implant. A second surgery to remove the implant may be needed in half of the patients due to irritation by the subcutaneously positioned implant. ${ }^{20,23}$ With lateral plates the distal metaphyseal screws must be unicortical to prevent articular surface penetration. This results in poor outcomes in elderly osteoporotic patients. A recent randomized observational study on surgically treated type B ankle fractures with minimal talar shift, has shown suboptimal outcomes with increased incidence of complications as compared to conservative management for the same. ${ }^{24}$

Literature evidence suggests that optimal outcomes can be achieved with conservative management of Weber type $B$ ankle fractures. ${ }^{24}$ The incidence of arthritis was similar in both surgically and conservatively managed patients $(2.8 \%$ in nonoperative vs $2.9 \%$ in operative group). ${ }^{21,22}$ In the current study, good functional outcomes was seen in $89 \%$ of patients who were conservatively managed study by Yde et al. ${ }^{22}$ In the present study, in all patients the fracture healed without residual medial clear space widening. In one patient there was a minimal talar shift of $<1 \mathrm{~mm}$ with an acceptable tibiofibular clear space of $5 \mathrm{~mm}$. This minimal shift was not quantified to any specific value to denote instability and this was asymptomatic as shown in the study by Sanders et al. ${ }^{6}$

In the present study, the mean AOFAS score was 93 at the final follow-up. The four fractures which were deemed unstable on external rotation stress view but found to be stable on weightbearing view had AOFAS score comparable with patients for whom both views were stable ( 92.7 vs 93 ). This aligns with the observations of Holmes et al. ${ }^{25}$ and Seidel et al. ${ }^{18}$

Only three (17\%) cases showed a delay in radiological healing, which was similarly observed by Sanders et al. with $20 \%$ cases showing delayed union in their study. ${ }^{6}$ Complications like residual ankle pain, nonunion, and early post-traumatic ankle arthritis were not observed in our study.

Limitations of the study:

The small sample size and the shorter follow-up of 14 months are the limitations of this study. However, as pain and functional outcomes are the most important criteria for assessment of clinical outcomes, the outcomes of treatment can be determined even without a long-term radiographic follow-up, as evidenced in the study by Finnan et al. ${ }^{26}$

\section{Conclusion}

Standalone WBR are feasible, and reliable in detecting deep deltoid ligament injury and instability, in Weber type B fractures of the lateral malleolus and thereby serve as a useful guide in deciding between nonoperative vs operative management. It is associated with better patient compliance. This method of assessment of ankle instability prevents overestimation of ankle instability, thereby avoiding unnecessary surgery in a stable fracture, as against previously used stress radiographic assessment methods. This substantially reduces the financial burden of surgery and avoids possible complications of surgery in a significant number of patients who would have been candidates for surgery had external rotation been used as a modality for detecting instability.

\section{References}

1. Daly PJ, Fitzgerald RH, Melton LJ, et al. Epidemiology of ankle fractures in Rochester, Minnesota. Acta Orthop Scand 1987;58(5):539-544. DOI: 10.3109/17453678709146395

2. Jensen SL, Andresen BK, Mencke S, et al. Epidemiology of ankle fractures: a prospective population-based study of 212 cases in Aalborg, Denmark. Acta Orthop Scand 1998;69(1):48-50. DOI: 10.3109/17453679809002356

3. Court-Brown CM, McBirnie J. Adult ankle fractures-an increasing problem? Acta Orthop 1998;69(1):43-47. DOI: 10.3109/17453679809002355

4. Yde J, Kristensen KD. Ankle fractures: supination-eversion fractures of stage IV. Primary and late results of operative and non-operative treatment. Acta Orthop Scand 1980;51(6):981-990. DOI: 10.3109/17453678008990904

5. Gougoulias N, Khanna A, Sakellariou A, et al. Supination-external rotation ankle fractures: stability a key issue. Clin Orthop Relat Res 2010;468(1):243-251. DOI: 10.1007/s11999-009-0988-2

6. Sanders DW, Tieszer C, Corbett B. Operative versus nonoperative treatment of unstable lateral malleolar fractures. J Orthop Trauma 2012;26(3):129-134. DOI: 10.1097/BOT.0b013e3182460837

7. Redfern DJ, Sauve PS, Sakellariou A. Investigation of incidence of superficial peroneal nerve injury following ankle fracture. Foot Ankle Int 2003;24(10):771-774. DOI: 10.1177/107110070302401006

8. Dawe EJC, Shafafy R, Quayle J, et al. The effect of different methods of stability assessment on fixation rate and complications in supination external rotation (SER) $2 / 4$ ankle fractures. Foot Ankle Surg 2015;21(2):86-90. DOI: 10.1016/j.fas.2014.09.01

9. Michelson JD, Magid D, McHale K. Clinical utility of a stability-based ankle fracture classification system. J Orthop Trauma 2007;21(5): 307-315. DOI: 10.1097/BOT.0b013e318059aea3

10. Hak DJ, Egol KA, Gardner MJ, et al. The "not so simple" ankle fracture: avoiding problems and pitfalls to improve patient outcomes. Instr Course Lect 2011;60:73-88. PMID: 21553763.

11. Michelsen JD, Ahn UM, Helgemo SL. Motion of the ankle in a simulated supination-external rotation fracture model. J Bone Joint Surg Am 1996;78(7):1024-1031. DOI: 10.2106/00004623-199607000-00006

12. DeAngelis NA, Eskander MS, French BG. Does medial tenderness predict deep deltoid ligament incompetence in supination-external rotation type ankle fractures? J Orthop Trauma 2007;21(4):244-247. DOI: 10.1097/BOT.0b013e3180413835

13. Koval KJ, Egol KA, Cheung Y, et al. Does a positive ankle stress test indicate the need for operative treatment after lateral malleolus fracture? A preliminary report. J Orthop Trauma 2007;21(7):449-455. DOI: 10.1097/BOT.0b013e31812eed25

14. Gougoulias N, Sakellariou A. When is a simple fracture of the lateral malleolus not so simple? Bone Joint J 2017;99-B(7):851-855. DOI: 10.1302/0301-620X.99B7.BJJ-2016-1087.R1

15. Weber M, Burmeister H, Flueckiger G, et al. The use of weightbearing radiographs to assess the stability of supination- external rotation fractures of the ankle. Arch Orthop Trauma Surg 2010;130(5):693-698. DOI: 10.1007/s00402-010-1051-1

16. Hoshino CM, Nomoto EK, Norheim EP, et al. Correlation of weightbearing radiographs and stability of stress positive ankle fractures. Foot Ankle Int 2012;33(2):92-98. DOI: 10.3113/FAl.2012.0092

17. Schock HJ, Pinzur M, Manion L, et al. The use of gravity or manualstress radiographs in the assessment of supination-external rotation fractures of the ankle. J Bone Joint Surg Br 2007;89(8):1055-1059. DOI: 10.1302/0301-620X.89B8.19134

18. Seidel A, Krause F, Weber M. Weightbearing vs gravity stress radiographs for stability evaluation of supination-external rotation fractures of the ankle. Foot Ankle Int 2017;38(7):736-744. DOI: $10.1177 / 1071100717702589$ 
19. McConnell T, Creevy W, Tornetta 3rd P. Stress examination of supination external rotation-type fibular fractures. J Bone Joint Surg Am 2004;86(10):2171-2178. DOI: 10.2106/00004623-20041000000007

20. Anderson SA, Li X, Franklin $\mathrm{P}$, et al. Ankle fractures in the elderly: initial and long-term outcomes. Foot Ankle Int 2008;29(12):1184-1188. DOI: 10.3113/FAI.2008.1184

21. Bauer M, Jonsson K, Nilsson B. Thirty-year follow-up of ankle fractures. Acta Orthop Scand 1985;56(2):103-106. DOI: 10.3109/17453678508994329

22. Yde J, Kristensen KD. Ankle fractures: supination-eversion fractures stage II. Primary and late results of operative and nonoperative treatment. Acta Orthop Scand 1980;51(4):695-702. DOI: $10.3109 / 17453678008990863$
23. McKenna PB, O'shea K, Burke T. Less is more: lag screw only fixation of lateral malleolar fractures. Int Orthop 2007;31(4):497-502. DOI: 10.1007/s00264-006-0216-6

24. Mittal R, Harris IA, Adie $S$, et al. Surgery for type B ankle fracture treatment: a Combined Randomised and Observational Study (CROSSBAT). BMJ Open 2017;7:e013298. DOI: 10.1136/bmjopen2016-013298

25. Holmes JR, Acker WB II, Murphy JM, et al. A novel algorithm for isolated Weber $B$ ankle fractures: a retrospective review of 51 nonsurgically treated patients. J Am Acad Orthop Surg 2016;24(9):645-652. DOI: 10.5435/JAAOS-D-16-00085

26. Finnan R, FunkL, Pinzur MS, et al. Health related quality of life in patients with supination-external rotation stage IV ankle fractures. Foot Ankle Int 2005;26(12):1038-1041. DOI: 10.1177/107110070502601207 